\title{
Populações de oligoquetos (Annelida: Oligochaeta) em um Latossolo Vermelho submetido a sistemas de uso do solo
}

\author{
Population of earthworm (Annelida: Oligochaeta) in a Hapludox under soil used systems
}

\author{
Rogério Ferreira da Silva ${ }^{1}$ Adriana Maria de Aquino² ${ }^{2}$ Fábio Martins Mercante $^{3}$ \\ Maria de Fátima Guimarães ${ }^{4}$
}

\begin{abstract}
O trabalho teve como objetivo avaliar a dinâmica RESUMO da população de oligoquetos edáficos em sistemas de manejo agrícola e pecuário, tendo, como referência, uma área sob vegetação nativa. O trabalho foi conduzido no município de Dourados, MS, num solo classificado como Latossolo Vermelho distroférrico típico, sob sistema com preparo convencional $(S C)$, plantio direto (SPD), integração lavoura/pecuária (SILP), pastagem contínua (PC) e sistema natural (SN). As amostragens foram realizadas nas safras de verão e inverno, no período de 2000 a 2003. Os sistemas SPD, SILP e PC favoreceram o desenvolvimento e estabelecimento da população dos oligoquetos edáficos. O sistema natural apresentou uma alta população de oligoquetos edáficos, sendo a grande maioria constituída pelos organismos da família Enchytraeidae. Dentre os sistemas produtivos, o SPD favoreceu a ocorrência dessa família.
\end{abstract}

Palavras-chave: manejo do solo, densidade populacional, oligoquetos.

\section{ABSTRACT}

This paper objective was to evaluate earthworm population dynamics in agricultural and livestock systems using as reference an area under native vegetation. The work was carried out in Dourados, MS, in a Typic Hapludox soil under conventional soil tillage (CT), no-tillage (NT), cropping/livestock integration (CLI), a continuous pasture (CP), and a natural system (NS). Samples were taken during summer and winter cropping seasons between 2000 and 2003. SPD, SILP, and PC systems benefited the development and establishment of earthworm population. NS system showed the largest earthworm population, being the majority of organisms from Enchytraeidae family. Among the systems, SPD also benefited the occurrence of organisms from Enchytraeidae family.

Key words: systems, population density, earthworms.

Os oligoquetos edáficos são denominados “engenheiros do ecossistema”, pois suas atividades levam à criação de estruturas biogênicas (galerias e coprólitos), que modificam as propriedades físicas dos solos onde vivem e a disponibilidade de recursos para outros organismos (JONES et al., 1994). Através das suas ações mecânicas no solo, contribuem para formação de agregados estáveis, que podem proteger parte da matéria orgânica de uma mineralização rápida, e constituem, também, uma reserva de nutrientes potencialmente disponíveis para as plantas (LAVELLE \& SPAIN, 2001). Portanto, esse grupo de organismos influencia, de maneira direta e indireta, os diversos processos físicos, químicos e biológicos do solo (ABRAHAMSEN, 1972; LAVELLE, 1997). Dentre esses processos, destacam-se a estabilidade estrutural, que inclui a formação de macro e microagregados; o aumento de porosidade, aeração e condutividade hidráulica; a reciclagem de carbono e nutrientes; além de estimular a atividade da microfauna e da fauna do solo,

${ }^{1}$ Programa de Pós-gradução em Agronomia, Universidade Estadual de Londrina (UEL), Londrina, PR, Brasil. Embrapa Agropecuária Oeste, Br 163 Km 253, Caixa Postal 661, 79804-970, Dourados, MS. E-mail: rogerio@cpao.embrapa.br. Autor para correspondência ${ }^{2}$ Embrapa Agrobiologia, Seropédica, RJ, Brasil.

${ }^{3}$ Embrapa Agropecuária Oeste, Dourados, MS, Brasil.

${ }^{4}$ UEL, Centro de Ciências Agrárias, Londrina, PR, Brasil. 
promovendo melhor desenvolvimento das plantas (LAVELLE \& SPAIN, 2001).

Além das variações sazonais de temperatura, umidade, tipo de solo e a qualidade de matéria orgânica, o grau de intensidade das atividades antrópicas são determinantes na dinâmica de população de oligoquetos edáficos. Segundo TANCK et al. (2000), as flutuações populacionais dos oligoquetos edáficos em áreas de cultivos dependem dos sistemas de manejo. A não-mobilização do solo e o aumento da matéria orgânica determinam, ao longo do tempo, um ambiente propício para a atividade e estabelecimento das minhocas (AMARAL et al., 2004). Sistemas conservacionistas, com a manutenção dos resíduos culturais na superfície do solo, agem positivamente no equilíbrio populacional durante as estações do ano (NUUTINEN, 1992). O presente trabalho teve como objetivo avaliar a dinâmica da população de oligoquetos edáficos em sistemas de manejo agrícola e pecuário, tendo, como referência, uma área sob vegetação nativa.

Os estudos foram conduzidos no período de 2000 a 2003, no campo experimental da Embrapa Agropecuária Oeste, município de Dourados-MS (22 14' $00^{\prime}$ ' S e 54 49'00” W), em área de Latossolo Vermelho distroférrico típico, com teores médios de 700 $\mathrm{g} \mathrm{kg}^{-1}$ de argila. O clima da região, segundo a classificação de Köppen, enquadra-se no tipo climático Cwa, definido como clima temperado com inverno seco e verão chuvoso (AMARAL et al., 2000).

Os sistemas de manejo avaliados no estudo foram implementados em 1995, com sistemas intensivos de produção, sendo caracterizados como: 1) sistema convencional (SC) - monocultivo de soja no verão e de aveia no outono/inverno, onde o solo foi preparado com grades de disco e herbicidas residuais foram aplicados em pré-emergência; 2) sistema plantio direto (SPD) - cultivo em plantio direto, com rotação de culturas, tendo-se no verão a seqüência soja/soja/ milho e no outono/inverno aveia/trigo/nabo forrageiro.

No SPD, foram empregados herbicidas pósemergentes e todas as tecnologias disponíveis para a região, como manejo integrado de pragas, visando obter elevados rendimentos de grãos e reduzir as perdas do sistema; 3) sistema integrado lavoura/pecuária (SILP) -soja/aveia, conduzidas em plantio direto, rotacionadas, a cada dois anos, com pastagem (Brachiaria decumbens Stapf); 4) pastagem contínua (PC) - consiste em pastagem permanente com Brachiaria decumbens, conduzida em sistema de pastejo contínuo e 5) sistema natural (SN) - fragmento de vegetação de Cerrados, contígua à área experimental, utilizada como referência da condição original do solo.
As amostragens foram realizadas em seis épocas: dez/00, jun/01, jan/02, jun/02, jan/03 e jul/03, correspondentes a três safras de verão e três de inverno. Em cada sistema, foram amostrados cinco monólitos de solo com 0,25 x 0,25m de largura e 0,30m de profundidade, ao longo de um transecto, distando-se cerca de 30m entre os pontos de avaliação, de acordo com ANDERSON \& INGRAM (1993). Os organismos foram extraídos manualmente e armazenados numa solução de formol 4\%. No laboratório, com auxílio de uma lupa binocular, procedeu-se à identificação e contagem dos organismos. Dentre os oligoquetos, a família Enchytraeidae foi avaliada separadamente e as demais foram agrupadas em outros Oligochaeta. A identificação dos organismos seguiu o procedimento descrito em CURSO INTERNACIONAL...(2004). O solo coletado em todas as épocas de amostragens foi caracterizado quimicamente pelo $\mathrm{pH}, \mathrm{P}, \mathrm{K}^{+}, \mathrm{Ca}^{++}, \mathrm{Mg}^{++}$, $\mathrm{Al}^{+++}$, soma de bases (V) e matéria orgânica (MO) (Tabela 1).

Os dados de densidade populacional de oligoquetos edáficos (x), dada a sua heterogeneidade, foram transformados em $\log (x+1)$ e submetidos à análise de variância. Utilizou-se o delineamento inteiramente casualizado em esquema fatorial (5 x 6), com cinco sistemas intensivos de produção para o primeiro fator e em seis épocas de avaliação, para o segundo fator, sendo as médias comparadas pelo teste de Duncan, ao nível de 5\%. Efetuou-se o teste de coeficiente de correlação de Pearson (r) para analisar a relação entre a matéria orgânica do solo e os dados de população de Enchytraeidae e outros oligoquetos.

Verificou-se que houve efeito significativo $(\mathrm{P}<0,05)$ da interação entre os sistemas avaliados e as épocas de amostragem sobre os valores de densidade de oligoquetos, corroborando com as observações de CHAN (2001), de que este comportamento, possivelmente, foi proporcionado pelas variações sazonais de temperatura e umidade do solo. Observouse que os valores de densidade populacional nos sistemas cultivados foram muito acentuados entre as seis épocas de avaliação (Tabela 2).

De acordo com os resultados apresentados na tabela 2, observou-se que o sistema natural (SN), em todas as épocas de avaliação, apresentou a maior densidade populacional de oligoquetos, diferindo dos demais sistemas. Esta maior ocorrência pode estar relacionada com as condições edáficas mais favoráveis, especialmente em relação às características químicas do solo (Tabela 1). Além disso, pode ter sido beneficiada pela variabilidade de microhabitats e maior oferta de recursos proporcionada pela diversidade de vegetação da mata. Em geral, os sistemas sob plantio direto (SPD), 
Tabela 1 - Caracterização química de solo na profundidade 0,0-0,30m sob sistema convencional (SC), plantio direto (SPD), integração lavoura/pecuária (SILP), pastagem contínua (PC) e sistema natural (SN), Dourados, MS.

\begin{tabular}{|c|c|c|c|c|c|c|}
\hline Características & Época & SC & SPD & SILP & PC & $\mathrm{SN}$ \\
\hline \multirow{6}{*}{$\mathrm{pH}\left(\mathrm{H}_{2} \mathrm{O}\right)$} & $\mathrm{dez} / 00$ & 5,1 & 5,2 & 5,2 & 5,7 & 5,9 \\
\hline & jun/01 & 5,2 & 5,2 & 5,1 & 5,5 & 5,9 \\
\hline & jan/02 & 5,0 & 5,3 & 5,2 & 5,4 & 6,0 \\
\hline & jun/02 & 5,1 & 5,4 & 5,2 & 5,5 & 6,2 \\
\hline & jan/03 & 5,3 & 5,5 & 5,5 & 5,5 & 6,2 \\
\hline & jul/03 & 5,4 & 5,5 & 5,3 & 5,7 & 5,9 \\
\hline \multirow{6}{*}{$\mathrm{P}\left(\mathrm{mg} \mathrm{dm}^{-3}\right)$} & dez/00 & 9,2 & 17,6 & 6,2 & 3,1 & 1,5 \\
\hline & jun/01 & 9,9 & 15,8 & 7,7 & 3,0 & 1,4 \\
\hline & jan/02 & 14,2 & 16,6 & 9,9 & 5,5 & 2,2 \\
\hline & jun/02 & 11,4 & 16,1 & 9,8 & 4,4 & 1,7 \\
\hline & jan/03 & 13,5 & 16,4 & 8,8 & 3,0 & 2,3 \\
\hline & jul/03 & 13,1 & 20,6 & 6,7 & 2,9 & 1,5 \\
\hline \multirow{6}{*}{$\mathrm{K}^{+}\left(\mathrm{cmol}_{\mathrm{c}} \mathrm{dm}^{-3}\right)$} & $\mathrm{dez} / 00$ & 0,3 & 0,4 & 0,2 & 0,4 & 0,5 \\
\hline & jun/01 & 0,4 & 0,4 & 0,2 & 0,3 & 0,5 \\
\hline & jan/02 & 0,5 & 0,3 & 0,3 & 0,3 & 0,4 \\
\hline & jun/02 & 0,4 & 0,3 & 0,2 & 0,3 & 0,5 \\
\hline & jan/03 & 0,4 & 0,4 & 0,2 & 0,4 & 0,8 \\
\hline & jul/03 & 0,4 & 0,4 & 0,2 & 0,4 & 0,4 \\
\hline \multirow{6}{*}{$\mathrm{Ca}^{++}\left(\mathrm{cmol}_{\mathrm{c}} \mathrm{dm}^{-3}\right)$} & $\operatorname{dez} / 00$ & 3,1 & 4,0 & 2,8 & 4,1 & 10,4 \\
\hline & jun/01 & 3,4 & 3,9 & 2,9 & 3,3 & 9,5 \\
\hline & jan/02 & 2,7 & 3,8 & 3,1 & 3,7 & 9,5 \\
\hline & jun/02 & 2,8 & 3,6 & 2,6 & 3,0 & 9,4 \\
\hline & jan/03 & 3,1 & 3,8 & 3,2 & 2,9 & 9,5 \\
\hline & jul/03 & 3,9 & 3,8 & 3,2 & 4,4 & 8,8 \\
\hline \multirow{6}{*}{$\mathrm{Mg}^{++}\left(\mathrm{cmol}_{\mathrm{c}} \mathrm{dm}^{-3}\right)$} & $\operatorname{dez} / 00$ & 1,2 & 1,7 & 1,2 & 1,8 & 2,9 \\
\hline & jun/01 & 1,1 & 1,5 & 1,2 & 1,7 & 2,7 \\
\hline & jan/02 & 1,0 & 1,7 & 1,4 & 2,0 & 2,9 \\
\hline & jun/02 & 1,0 & 1,5 & 1,5 & 2,0 & 2,4 \\
\hline & jan/03 & 1,4 & 1,8 & 1,8 & 2,0 & 2,9 \\
\hline & jul/03 & 1,8 & 1,9 & 1,6 & 2,4 & 2,7 \\
\hline \multirow{6}{*}{$\mathrm{Al}^{+++}\left(\mathrm{cmol}_{\mathrm{c}} \mathrm{dm}^{-3}\right)$} & $\operatorname{dez} / 00$ & 1,3 & 0,7 & 0,8 & 0,2 & 0,1 \\
\hline & jun/01 & 1,0 & 0,7 & 1,0 & 0,3 & 0,1 \\
\hline & jan/02 & 1,3 & 0,6 & 0,8 & 0,5 & 0,0 \\
\hline & jun/02 & 1,1 & 0,6 & 0,8 & 0,4 & 0,1 \\
\hline & jan/03 & 0,9 & 0,5 & 0,6 & 0,4 & 0,0 \\
\hline & jul/03 & 0,7 & 0,5 & 0,9 & 0,2 & 0,1 \\
\hline \multirow{6}{*}{ V (\%) } & $\mathrm{dez} / 00$ & 29,8 & 43,9 & 33,5 & 51,6 & 69,1 \\
\hline & jun/01 & 30,5 & 38,3 & 30,3 & 44,0 & 67,0 \\
\hline & jan/02 & 27,0 & 40,0 & 33,8 & 40,1 & 69,4 \\
\hline & jun/02 & 29,5 & 42,4 & 32,2 & 44,3 & 68,4 \\
\hline & jan/03 & 34,7 & 43,7 & 39,8 & 41,8 & 70,9 \\
\hline & jul/03 & 42,2 & 46,2 & 38,5 & 54,5 & 64,4 \\
\hline \multirow{6}{*}{ MO $\left(\mathrm{g} \mathrm{kg}^{-1}\right)$} & dez/00 & 25,9 & 27,5 & 28,0 & 31,3 & 45,9 \\
\hline & jun/01 & 27,7 & 26,4 & 26,8 & 28,6 & 53,0 \\
\hline & jan/02 & 24,7 & 26,4 & 26,4 & 29,8 & 46,2 \\
\hline & jun/02 & 27,2 & 24,8 & 26,0 & 29,3 & 46,1 \\
\hline & jan/03 & 25,7 & 26,8 & 29,1 & 30,8 & 57,3 \\
\hline & jul/03 & 23,7 & 25,1 & 27,1 & 30,5 & 46,7 \\
\hline
\end{tabular}

Ciência Rural, v.36, n.2, mar-abr, 2006. 
Tabela 2 - Densidade populacional média (ind $\mathrm{m}^{-2}$ ) de oligoquetos edáficos, profundidade de 0,0-0,30m, sob sistema convencional (SC), plantio direto (SPD), integração lavoura/pecuária (SILP), pastagem contínua (PC) e sistema natural (SN), Dourados, MS.

\begin{tabular}{|c|c|c|c|c|c|c|}
\hline \multirow{2}{*}{ Sistemas } & \multicolumn{6}{|c|}{ Época de avaliação } \\
\hline & $\operatorname{dez} / 00$ & jun/01 & jan/02 & jun/02 & jan/03 & jul/03 \\
\hline & \multicolumn{6}{|c|}{ Enchytraeidae } \\
\hline SC & 0Bb* & $13 \mathrm{Ca}$ & $\mathrm{OBb}$ & $0 \mathrm{Cb}$ & $0 \mathrm{Cb}$ & $0 \mathrm{Bb}$ \\
\hline SPD & 123Aa & 49Bа & $3 \mathrm{Bb}$ & $2 \mathrm{Cb}$ & $4 \mathrm{Cb}$ & $0 \mathrm{Bb}$ \\
\hline SILP & $2 \mathrm{Ba}$ & 0Da & 0Ba & $27 \mathrm{Ba}$ & 0Ca & $2 \mathrm{Ba}$ \\
\hline PC & $\mathrm{OBb}$ & $3 \mathrm{CDb}$ & $6 \mathrm{Bb}$ & $3 \mathrm{Cb}$ & 29Ba & $\mathrm{OBb}$ \\
\hline \multirow[t]{2}{*}{ SN } & $240 \mathrm{Ab}$ & 870Aa & 3347Aa & 2813Aa & 1667Aa & $509 \mathrm{Ab}$ \\
\hline & \multicolumn{6}{|c|}{ Outros Oligochaeta } \\
\hline SC & $13 \mathrm{Cb}$ & $70 \mathrm{Ba}$ & 0Cc & 6Cbc & 0Bc & 0Сc \\
\hline SPD & 31Bbc & 195Aa & 29Bbc & $46 \mathrm{Bb}$ & $26 \mathrm{Abc}$ & $16 \mathrm{Bc}$ \\
\hline SILP & 24Bbc & 264Aa & $26 \mathrm{Bbc}$ & 91Bab & 48Abc & $6 \mathrm{Bc}$ \\
\hline PC & 29Ba & $90 \mathrm{ABa}$ & 93АВа & $77 \mathrm{Ba}$ & 93Aa & 58Aba \\
\hline \multirow[t]{2}{*}{ SN } & 48Aa & 106Aa & $147 \mathrm{Aa}$ & 179Aа & 93Aa & 70Aa \\
\hline & \multicolumn{6}{|c|}{ Total de oligoquetos } \\
\hline SC & $13 \mathrm{Cb}$ & 83Ca & 0Cc & 6Cbc & 0Dc & 0Сc \\
\hline SPD & $154 \mathrm{ABb}$ & 244Ba & 32Bbc & $48 \mathrm{Bb}$ & $30 \mathrm{Cbc}$ & $16 \mathrm{Bc}$ \\
\hline SILP & 26Bbc & 264Ba & 26Bbc & 118Bab & 48Cbc & $8 \mathrm{Bc}$ \\
\hline PC & $29 B \mathrm{a}$ & 93BCa & 99Bа & 80Ва & 122BCa & 58Ba \\
\hline $\mathrm{SN}$ & 288Ab & 976Аа & 3494Aa & 2992Аа & 1760Aa & 579Ab \\
\hline
\end{tabular}

*Para a análise estatística, os valores foram transformados por log $(\mathrm{x}+1)$. Médias grafadas com a mesma letra maiúscula, na coluna, e minúscula, na linha, não diferem entre si pelo teste de Duncan a 5\% de significância.

integração lavoura/pecuária (SILP) e pastagem contínua (PC) apresentaram valores de densidade populacional semelhantes entre si e superiores ao sistema convencional (SC). Isto se deve, principalmente, ao fato de o manejo em plantio direto promover acúmulo de material orgânico na superfície do solo e pequena movimentação mecânica (KLUTHCOUSKI \& AIDAR, 2003). As pastagens cultivadas continuamente apresentam grande densidade de raízes em constante renovação e exsudatos radiculares, conseqüentemente, maior disponibilidade de matéria orgânica no solo. Esses aspectos favorecem as condições para o desenvolvimento e estabelecimento dos oligoquetos edáficos (CHAN, 2001).

Os enquitreídeos foram mais abundantes na área de mata, indicando que houve favorecimento pelo maior aporte de matéria orgânica (Tabelas 1 e 2). Entretanto, dentre os sistemas de produção, o SPD nas épocas de dez/00 e ju/01, o SILP na época jun/02 e o PC na época jan/03 foram os que apresentaram as maiores densidades populacionais de Enchytraeidae, diferindo dos demais sistemas (Tabela 2). Verificou-se a presença desses organismos no SPD praticamente em todas as épocas avaliação, demonstrando que a rotação/sucessão de culturas aumenta o espectro de exsudatos e aporte de restos culturais no sistema, interferindo na qualidade da matéria orgânica e, conseqüentemente, favorecendo as condições para a ocorrência desse grupo. Foi observada correlação positiva $(r=0,72 ; P<0,05)$ entre o conteúdo de matéria orgânica do solo e a densidade de enquitreídeos, confirmando a importância da matéria orgânica como fonte de alimento para estes organismos.

Existe pouca informação sobre o papel dos Enchytraeidae nos processos que ocorrem no solo, entretanto, acredita-se que podem ter uma ação significativa sobre a ciclagem de nutrientes e no processo de decomposição, pela sua capacidade em revolver a matéria orgânica no solo, digerir de maneira seletiva microrganismos e pela dispersão de esporos (VAN VLIET et al., 1995). Pelas razões expostas e pelos resultados obtidos neste estudo, este grupo constituise numa premissa indispensável para pesquisas futuras, podendo corroborar como ferramenta na sua indicação como bioindicador de qualidade do solo.

Para outros Oligochaeta, observou-se dentre os sistemas SPD, SILP e PC certa similaridade e 
diferenças em relação ao sistema convencional (SC) (Tabela 2). Não foi observada boa correlação positiva $(r=0,38 ; P<0,05)$ entre a densidade de organismos e os teores de matéria orgânica, indicando que essas populações de oligoquetos eram dependentes de outros fatores como temperatura, umidade e qualidade da matéria orgânica (LEE, 1985).

Conclui-se que os sistemas SPD, SILP e PC favoreceram o desenvolvimento e estabelecimento da população dos oligoquetos edáficos. O sistema natural apresentou uma alta população de oligoquetos edáficos, sendo a grande maioria constituída pelos organismos da família Enchytraeidae. Dentre os sistemas produtivos, o SPD favoreceu a ocorrência dessa família, praticamente em todas a fases de avaliação.

\section{REFERÊNCIAS}

ABRAHAMSEN, G. Ecological study of Enchytraeidae (Oligochaeta) in Norwegian coniferous forest soils. Pedobiologia, v.12, p.26-82, 1972.

AMARAL, A.S. et al. Movimentação de partículas de calcário no perfil de um Cambissolo em plantio direto. Revista Brasileira Ciência do Solo, v.28, n.2, p.359-367, 2004.

AMARAL, J.A.M. et al. Levantamento semidetalhado dos solos do campo experimental de Dourados, da Embrapa Agropecuária Oeste, Município de Dourados, MS. Dourados: Embrapa Agropecuária Oeste; Rio de Janeiro: Embrapa Solos, 2000. 68p. (Embrapa Agropecuária Oeste. Documentos, 22; Embrapa Solos. Documentos, 15).

ANDERSON, J.M.; INGRAM, J.S.I. Tropical soil biology and fertility: a handbook of methods. 2.ed. Wallingford: CAB, 1993. 221p.

CHAN, K.Y. An overview of some tillage impacts on earthworm population abundance and diversity - implications for functioning in soils. Soil \& Tillage Research, v.57, p.179191, 2001.

CURSO INTERNACIONAL DE TAXONOMIA DE MINHOCAS, 2., 2004, Londrina. ELAETAO. Londrina: Embrapa Soja: Universidade Estadual de Londrina, 2004. Não paginado.

JONES, C.G. et al. Organisms as ecosystem engineers. Oikos, v.69, p.373-386, 1994.

KLUTHCOUSKI, J.; AIDAR, H. Uso da integração lavourapecuária na recuperação de pastagens degradas. In: KLUSTHCOUSKI, J. et al. Integração lavoura-pecuária. Santo Antônio de Goiás: Embrapa Arroz e Feijão, 2003. Cap.7, p.185-223.

LAVELLE, P. Faunal activities and soil processes: adaptive strategies that determine ecossystem function. Advances in Ecological Research, v.27, p.93-132, 1997.

LAVELLE, P.; SPAIN, A.V. Soil ecology. Dordrecht: Kluwer Academic, 2001. 654p.

NUUTINEN, V. Earthworm community response to tillage and residue management on different soil types in southern Finland. Soil \& Tillage Research, v.23, p.221-239, 1992.

LEE, K.E. Earthworms their ecology and relationships with soils and land use. Canberra: Academic, 1985. 411p.

TANCK, B.C.B. et al. Influência de diferentes sistemas de uso e manejo dos solos sobre a flutuação populacional de Oligochaeta edáfico Amynthas spp. Revista Brasileira de Ciência do Solo, v.24, p.409-415, 2000.

VAN VLIET, P.C.J. et al. Population dynamics and functional roles of Enchytraeidae (Oligochaeta) in hardwood forest and agricultural ecosystems. Plant and Soil, v.170, p.199-207, 1995. 